\title{
NUMERICAL SOLUTION OF A FLOW INSIDE A LABYRINTH SEAL
}

\begin{abstract}
Jan ŠıMÁK ${ }^{*}$, Petr STRAKA, Jaroslav PELANT
Abstract: The aim of this study is a behaviour of a flow inside a labyrinth seal on a rotating shaft. The labyrinth seal is a type of a non-contact seal where a leakage of a fluid is prevented by a rather complicated path, which the fluid has to overcome. In the presented case the sealed medium is the air and the seal is made by a system of 20 teeth on a rotating shaft situated against a smooth static surface. Centrifugal forces present due to the rotation of the shaft create vortices in each chamber and thus dissipate the axial velocity of the escaping air. The structure of the flow field inside the seal is studied through the use of numerical methods. Three-dimensional solution of the Navier-Stokes equations for turbulent flow is very time consuming. In order to reduce the computational time we can simplify our problem and solve it as an axisymmetric problem in a two-dimensional meridian plane. For this case we use a transformation of the Navier-Stokes equations and of the standard k-omega turbulence model into a cylindrical coordinate system. A finite volume method is used for the solution of the resulting problem. A one-side modification of the Riemann problem for boundary conditions is used at the inlet and at the outlet of the axisymmetric channel. The total pressure and total density (temperature) are to be used preferably at the inlet whereas the static pressure is used at the outlet for the compatibility. This idea yields physically relevant boundary conditions. The important characteristics such as a mass flow rate and a power loss, depending on a pressure ratio (1.1 - 4) and an angular velocity (1000 - 15000 rpm) are evaluated.
\end{abstract}

\section{INTRODUCTION}

The aim of this study is to investigate a behaviour of a flow inside a labyrinth seal and evaluate its characteristics such as a mass flow rate and a power loss. The labyrinth seal is a type of a noncontact seal where a leakage of a fluid is prevented by a rather complicated path, which the fluid has to overcome. In the presented case, we study a seal attached to a rotating turbine shaft, which is a part of an experimental device. The sealed medium is an air and the labyrinth seal is made by a set of twenty teeth situated on the rotating shaft against a smooth static surface. Centrifugal forces present due to the rotation of the shaft create vortices in each chamber and thus dissipate the axial velocity of the escaping air.

\section{NUMERICAL MODEL}

The flow of the air through the seal is described by a system of the RANS equations combined with a $k-\omega$ turbulence model $[1,5]$. The form of the equations for a mean value variables is the following:

$$
\begin{aligned}
\frac{\partial \rho}{\partial t}+\operatorname{div}(\rho \mathbf{v}) & =0, \\
\frac{\partial \rho v_{i}}{\partial t}+\operatorname{div}\left(\rho v_{i} \mathbf{v}\right) & =\operatorname{div}(\mathcal{T})_{i}, \quad i=1,2,3, \\
\frac{\partial E}{\partial t}+\operatorname{div}(E \mathbf{v}) & =\operatorname{div}(\mathcal{T} \mathbf{v})-\operatorname{div} \mathbf{q} .
\end{aligned}
$$

We use a standard notation here, the stress tensor $\mathcal{T}$ is defined by

$$
\mathcal{T}=(-p+\lambda \operatorname{div} \mathbf{v}) \boldsymbol{I}+2 \mu \boldsymbol{D}
$$

\footnotetext{
*Výzkumný a zkušební letecký ústav, a.s., Beranových 130, 19905 Praha 9 - Letňany, Czech republic, simak@vzlu.cz
} 
where $\boldsymbol{D}$, a strain rate tensor, is given by $\boldsymbol{D}=1 / 2\left(\nabla \mathbf{v}+\nabla \mathbf{v}^{T}\right)$.

Taking into consideration the geometry of our problem, it is really natural to use cylindrical coordinates, which simplify the description of the problem. The Cartesian coordinates $(x, y, z)$ are then transformed to a new coordinate system $(h, r, \varphi)$,

$$
\begin{aligned}
h & =x, \\
r & =\sqrt{y^{2}+z^{2}}, \\
\varphi & =\arctan (z / y) .
\end{aligned}
$$

The inverse coordinate transformation can be, of course, expressed as

$$
\begin{aligned}
& x=h, \\
& y=r \cos \varphi, \\
& z=r \sin \varphi .
\end{aligned}
$$

A transformation of a vector will be of the form

$$
(u, v, w)=\left(u_{r}, v_{r} \cos \varphi-w_{r} \sin \varphi, v_{r} \sin \varphi+w_{r} \cos \varphi\right) .
$$

It is possible to show that the gradient expressed in cylindrical coordinates is of the form

$$
\nabla f=\left(\frac{\partial f}{\partial h}, \frac{\partial f}{\partial r}, \frac{1}{r} \frac{\partial f}{\partial \varphi}\right)
$$

and that the divergence of a vector in the new system is

$$
\operatorname{div} \mathbf{v}=\frac{\partial u_{r}}{\partial h}+\frac{\partial v_{r}}{\partial r}+\frac{v_{r}}{r}+\frac{1}{r} \frac{\partial w_{r}}{\partial \varphi} .
$$

The flow inside the seal can be assumed axisymmetric so the derivatives in the $\varphi$ direction can be neglected. Thus the third space variable is eliminated from the system and we can solve our problem in a two-dimensional plane section along the axis. In the following text, we will deal only with cylindrical coordinates. The notations $x_{i}$ and $v_{i}$ denote components of a space and a velocity vector, now with respect to the new coordinates.

After some rearrangements, the equations (1) - (3) can be written in the form of

$$
\begin{aligned}
\frac{\partial \rho}{\partial t}+\sum_{j=1}^{2} \frac{\partial \rho v_{j}}{\partial x_{j}}+\frac{\rho v_{2}}{x_{2}} & =0 \\
\frac{\partial \rho v_{i}}{\partial t}+\sum_{j=1}^{2} \frac{\partial\left(\rho v_{i} v_{j}+\delta_{i j} p\right)}{\partial x_{j}}+\left(1+\delta_{i 3}\right) \frac{\rho v_{i} v_{2}}{x_{2}}-\delta_{i 2} \frac{\rho v_{3}^{2}}{x_{2}} & =\sum_{j=1}^{2} \frac{\partial \hat{\tau}_{i j}}{\partial x_{j}}+\left(1+\delta_{i 3}\right) \frac{\hat{\tau}_{i 2}}{x_{2}}+\delta_{i 2} \frac{\hat{\tau}_{33}}{x_{2}} \\
\frac{\partial E}{\partial t}+\sum_{j=1}^{2} \frac{\partial(E+p) v_{j}}{\partial x_{j}}+\frac{(E+p) v_{2}}{x_{2}}= & \sum_{j=1}^{2} \frac{\partial}{\partial x_{j}}\left(\sum_{k=1}^{3} \hat{\tau}_{j k} v_{k}+\kappa\left(\frac{\mu}{\operatorname{Pr}}+\frac{\mu_{T}}{\operatorname{Pr}_{T}}\right) \frac{\partial e}{\partial x_{j}}\right)+ \\
& +\frac{1}{r}\left(\sum_{k=1}^{3} \hat{\tau}_{2 k} v_{k}+\kappa\left(\frac{\mu}{\operatorname{Pr}}+\frac{\mu_{T}}{\operatorname{Pr}_{T}}\right) \frac{\partial e}{\partial x_{2}}\right)
\end{aligned}
$$

The $k-\omega$ turbulence model is of the form

$$
\begin{gathered}
\frac{\partial \rho k}{\partial t}+\sum_{j=1}^{2} \frac{\partial \rho k v_{j}}{\partial x_{j}}+\frac{\rho k v_{2}}{x_{2}}=\hat{P}_{k}-\beta^{*} \rho \omega k+\sum_{j=1}^{2} \frac{\partial}{\partial x_{j}}\left(\left(\mu+\sigma_{k} \mu_{T}\right) \frac{\partial k}{\partial x_{j}}\right)+\frac{1}{r}\left(\mu+\sigma_{k} \mu_{T}\right) \frac{\partial k}{\partial x_{2}} \\
\frac{\partial \rho \omega}{\partial t}+\sum_{j=1}^{2} \frac{\partial \rho \omega v_{j}}{\partial x_{j}}+\frac{\rho \omega v_{2}}{x_{2}}=\hat{P}_{\omega}-\beta \rho \omega^{2}+\sum_{j=1}^{2} \frac{\partial}{\partial x_{j}}\left(\left(\mu+\sigma_{\omega} \mu_{T}\right) \frac{\partial \omega}{\partial x_{j}}\right)+\frac{1}{r}\left(\mu+\sigma_{\omega} \mu_{T}\right) \frac{\partial \omega}{\partial x_{2}} . \\
01087-\text { p.2 }
\end{gathered}
$$


The above mentioned system of partial differential equations is solved by an implicit finite volume method on a structured grid. As a boundary condition for an inlet part of the boundary, we prescribe a total temperature and a total pressure. On the outlet part of the boundary, a static pressure is prescribed. To obtain proper values of state variables on a boundary edge, located on the inlet or outlet boundary, a modified $1 \mathrm{~d}$ Riemann problem is solved. The formulation of this representation of boundary conditions can be found in [2].

\section{RESULTS}

The numerical method is validated on a smooth cylinder rotating around its axis and the computed moment is compared with values obtained by Theodorsen [4]. Even though the flow is not exactly axisymmetric due to the presence of instabilities in a boundary layer, the model is sufficiently precise for our purpose. Moreover, in cases with a pressure gradient present, these effects are diminished and thus do not spoil the results.

\subsection{VALIDATION ON A SMOOTH CYLINDER}

The problem of a drag of a revolving cylinder was extensively studied in the past and many experimental results can be found in the literature. The drag coefficient for a revolving cylinder, which is equal to a moment coefficient, is given by

$$
C_{D}=\frac{M}{q S r},
$$

where $M$ is a moment acting on the cylinder, $q$ is a dynamic pressure (defined by $q=1 / 2 \rho \omega^{2} r^{2}$ ), $S$ is a surface of the cylinder and $r$ is a radius. The symbol $\omega$ will denote an angular velocity. The theoretical drag coefficient for a turbulent flow can be expressed as a function of the Reynolds number $R\left(R=\rho \omega r^{2} / \mu\right)$,

$$
\frac{1}{\sqrt{C_{D}}}=-0.6+4.07 \log _{10}\left(R \sqrt{C_{D}}\right) .
$$

The Figure 1 shows a theoretical prediction and evaluated values for two cylinders, differing in a diameter $r$ and a length $l$. The comparison with measurements presented in [4] shows a quite good agreement.

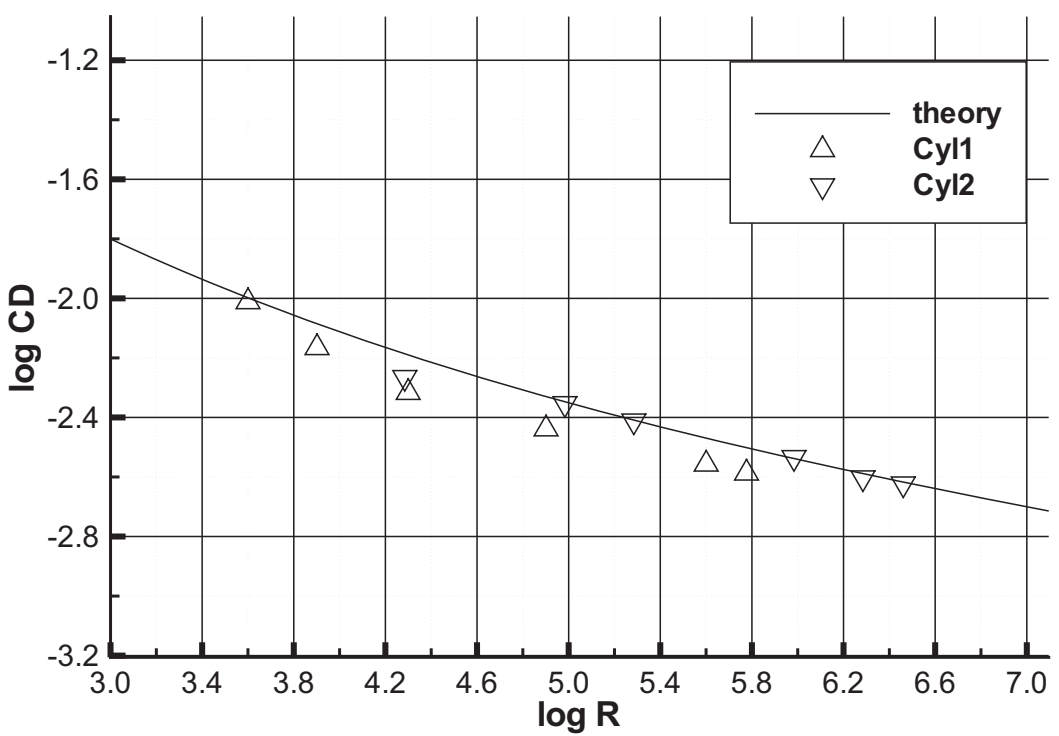

Figure 1: The drag coefficient on rotating cylinders, Cyl1: $r=0.0762, l=0.9144$, Cyl2: $r=0.1675, l=0.1178$ 


\subsection{ShAFT SEAL}

The rotating turbine shaft is sealed by a labyrinth seal, which is made of twenty teeth. The plane section is depicted in the Figure 2. The total length of the seal is $117 \mathrm{~mm}$ and the smallest gap is $0.5 \mathrm{~mm}$. The air is entering the seal through a narrow gap from the left and leaving it on the right side. Due to a large opening of the seal, there is a recirculation across the outlet boundary. As an inlet boundary condition, a total temperature $T_{0}=20^{\circ} \mathrm{C}$ and a total pressure $p_{0}$ in the range of $40-130 \mathrm{kPa}$ are prescribed. The results were computed for different back pressure ratios $p_{0} / p_{1}$ in the range of $1.1-4.0$ and for design regimes between 1000 - 15000 revolutions per minute.

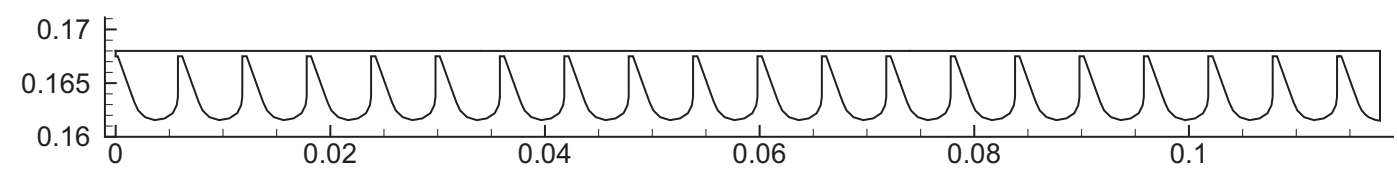

Figure 2: The geometry of a plane section of the shaft seal

The structure of the flow inside a cavity is shown in the Figure 3. The vortices are caused by centrifugal forces and by the geometry of the rotating lower wall. Also it is clearly seen a pressure drop between cavities.

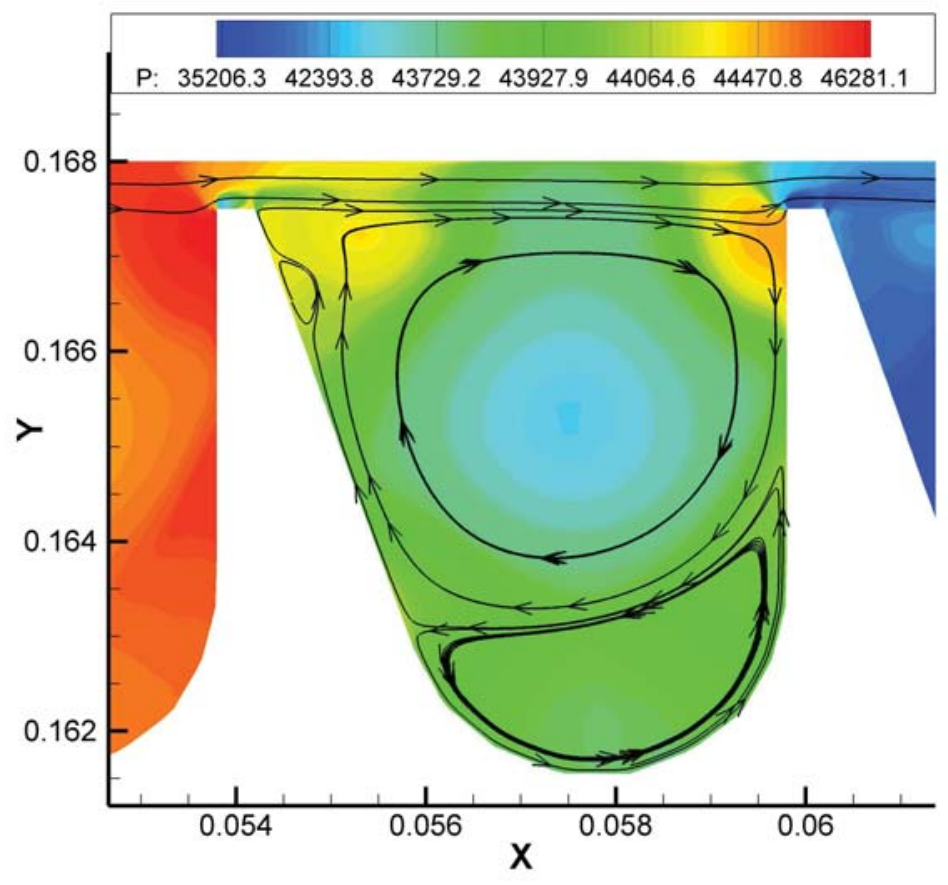

Figure 3: Pressure distribution in a cavity and streamlines

The graphs in the Figure 4 show a dependence between a moment acting on the shaft due to the flowing air and a back pressure ratio $p_{0} / p_{1}$. It is seen that increasing the back pressure ratio or increasing revolutions leads to a higher moment. Concerning the leakage of the air through the seal, increasing the back pressure ratio causes a higher mass flow rate. But increasing the angular speed decreases the leakage (Figure 5). When the back pressure ratio is high enough, the flow is chocked and the mass flow rate remains further constant. The last Figure 6 shows a relation between revolutions of the shaft and a mass flow rate and power losses. 

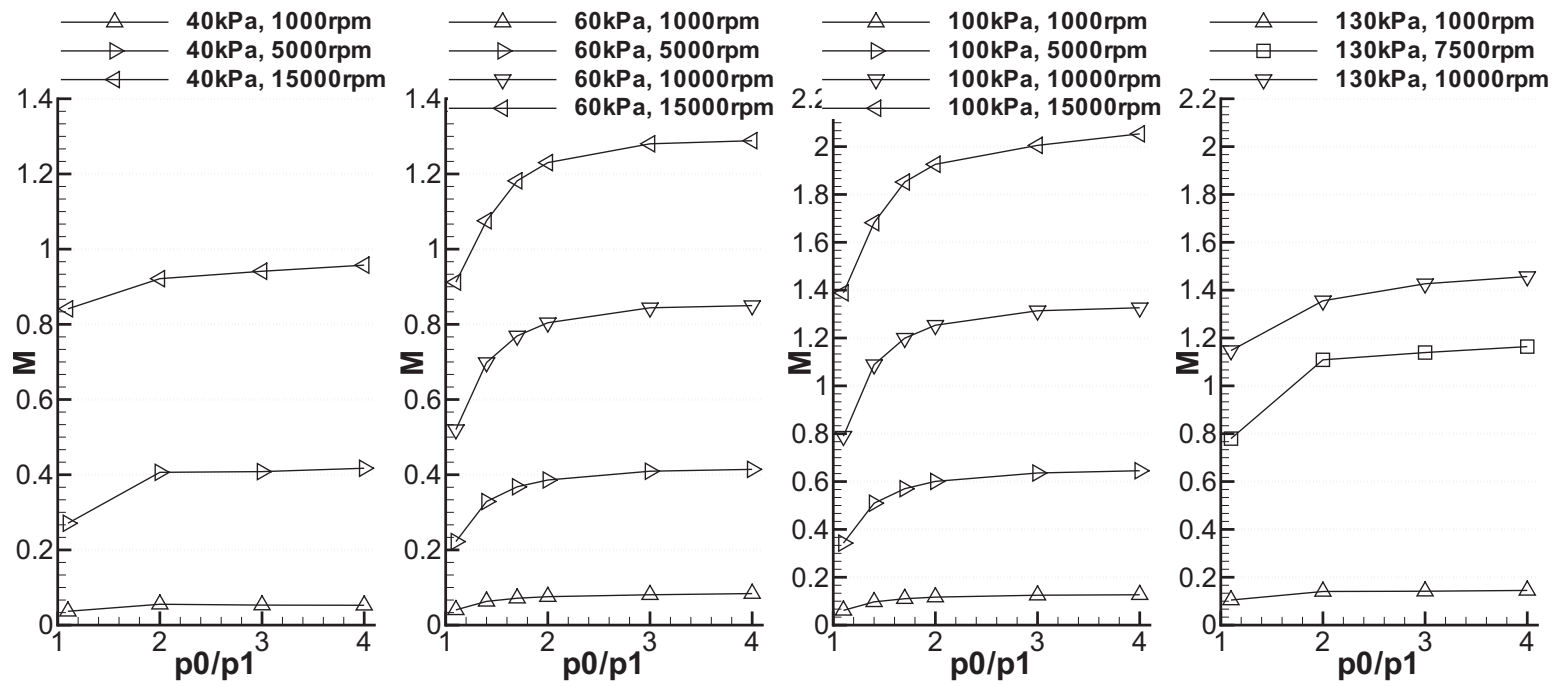

Figure 4: The moment $[\mathrm{Nm}]$ acting on the shaft as a function of the pressure ratio $p_{0} / p_{1}$
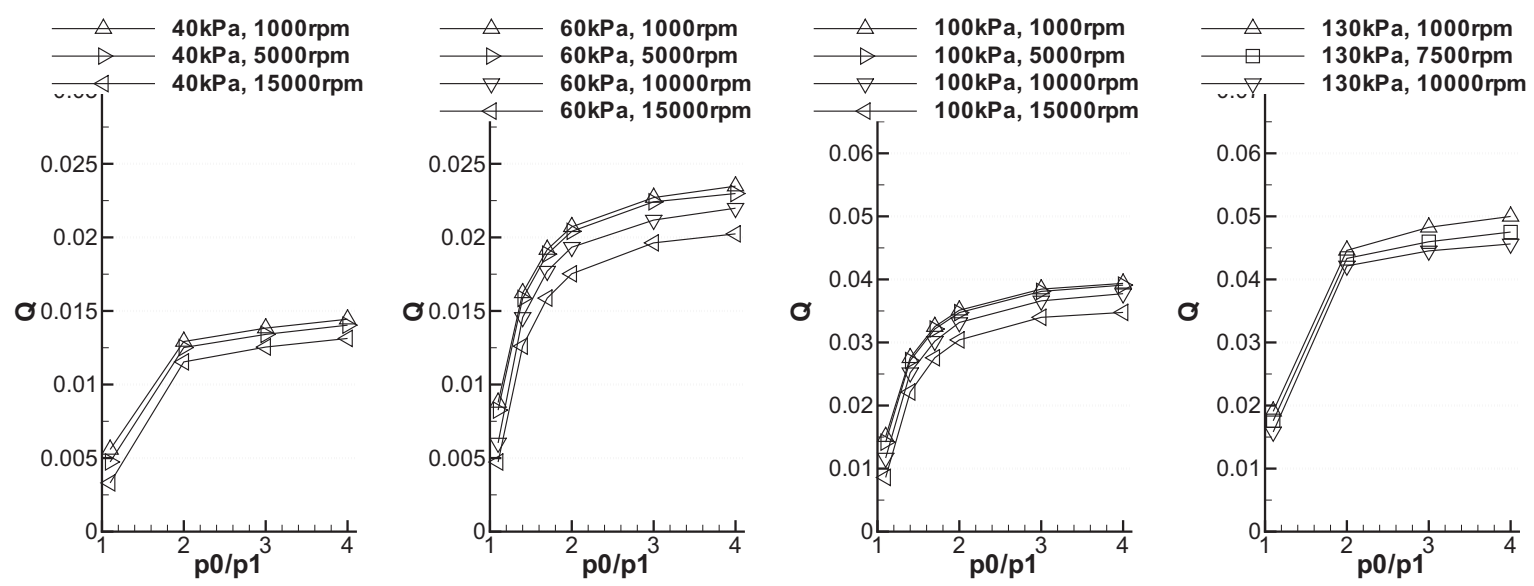

Figure 5: The leakage of the air $[\mathrm{kg} / \mathrm{s}]$ through the seal as a function of the pressure ratio $p_{0} / p_{1}$
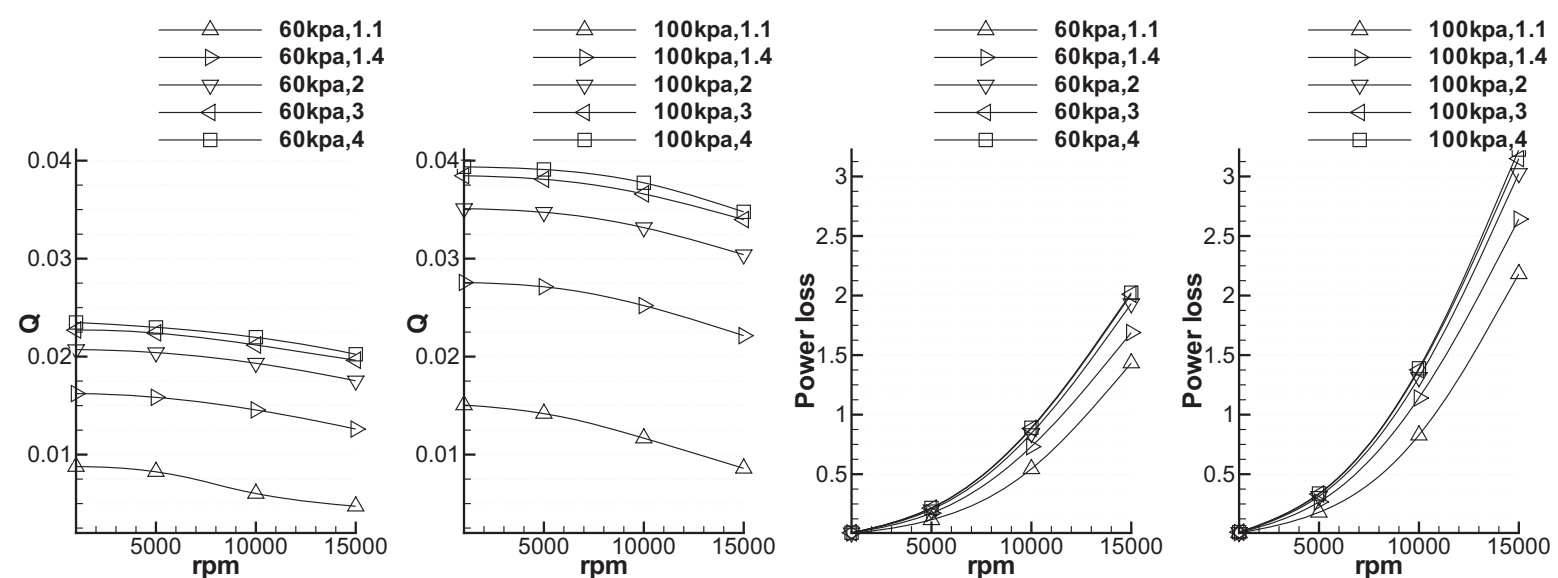

Figure 6: The dependence of the leakage of the air [kg/s] through the seal and of the power loss $[\mathrm{kW}]$ on revolutions, for different back pressure ratios 


\section{Discussion}

The mentioned method was used to solve a flow inside a labyrinth seal. The real problem was simplified to an axisymmetric problem, which is very close to the reality. The results are in accordance with the expectations. The advantage of the method is its speed and reasonable computer requirements. Nevertheless, it will be worth comparing obtained results with a full $3 \mathrm{~d}$ model in the future.

Another problem may be a propagation of a heat, especially in the case of a higher angular frequency. The air is heated by the moving wall and the temperature of the seal is rising. This problem requires a better treatment of boundary conditions on walls, including heat transfer to the surroundings. But in the assumed extent of regimes, this problem is not so noticeable.

\section{REFERENCES}

[1] Kok J.C.: Resolving the Dependence on Freestream Values for the $k-\omega$ Turbulence Model, AIAA Journal Vol. 38, No. 7, July 2000

[2] Kyncl M., Pelant J.: Applications of the Navier-Stokes Equations for 3d Viscous Laminar Flow for Symmetric Inlet and Outlet Parts of Turbine Engines with the Use of Various Boundary Conditions, VZLU Report R-3998, Prague, 2006

[3] Kyncl M., Pelant J.: Applications of the Navier-Stokes Equations for 3d Viscous, Compressible Laminar and Turbulent Flow in the Axis-symmetrical Channels as Parts of Turbine Engines with Steady or Moving Walls, VZLU Report R-4301, Prague, 2007

[4] Theodorsen T., Regier A.: Experiment on Drag of Revolving Disks, Cylinders, and Streamline Rods at High Speeds, NACA Report No. 793, 1944

[5] Wilcox D.C.: Turbulence Modeling for CFD, 2nd ed., DCW Industries Inc., 1998 